\title{
TEM Specimen Preparation for In Situ Heating Experiments Using FIB
}

\author{
Sriram Vijayan ${ }^{1}$, Mark Aindow ${ }^{1}$, Joerg. R. Jinschek ${ }^{2}$, Stephan Kujawa ${ }^{2}$ and Jens Greiser ${ }^{2}$ \\ ${ }^{1 .}$ Department of Materials Science and Engineering, Institute of Materials Science, University of \\ Connecticut, Storrs, CT, USA. \\ 2. FEI Company, Achtseweg Noord 5, Eindhoven, The Netherlands.
}

MEMS based devices have significantly improved our capability to capture the dynamic behavior of material inside the TEM at elevated temperatures and high spatial resolutions. The superior thermal stability of these MEMS based heating holders enables us to study a wide range of material systems ranging from nanoparticulate to bulk samples [1]. In situ investigations on nanoparticulate systems are relatively straightforward, but the study of bulk material systems is challenging due to complications in the preparation and the transfer of these specimens onto the MEMS chip. Previously, TEM specimens from bulk materials were prepared either by FIB milling an electron transparent fragment from an electropolished foil [2] or thinning down a thick lamella placed across the window of the MEMS chip with an ion beam [3]. In the former case, the site selectivity is limited to features from electron transparent regions of the electropolished foils, and the specimen is not mechanically robust so it can be damaged easily during the transfer onto the MEMS device. In the latter case, there are challenges in transferring the specimen onto the MEMS device, and the ion beam can damage the specimen and/or device during final thinning.

Here, we describe a novel specimen preparation protocol for the preparation and transfer of specimens from bulk samples onto a MEMS chip. Our work uses an FEI Helios Nanolab 460F1 FIB and an FEI, NanoEx-i/v heating holder, but the approach can be adapted readily for other FIBs and heating holders. This approach utilizes a specimen geometry with a thick layer surrounding the electron transparent region and a planar lower surface, which increase the mechanical stiffness, the area of the electron transparent region, and the contact area between the specimen and the micro-heater surface on the MEMS chip. The method involves a stage block with a flat top surface for mounting the bulk sample, and an inclined surface facing away from the ion beam for the MEMS chip. This arrangement minimizes the ion beam flux on the MEMS chip during milling. The stage block allows the use of conventional FIB lift out and milling procedures to prepare the specimen from the bulk sample (Figures 1(a-e)). The specimen transfer is initiated by separating the specimen from the $\mathrm{Cu}$-grid (Figure 1(f)) and tilting the bulk stage to allow for the accurate and reliable placement of the specimen across the MEMS window by imaging simultaneously using the electron and ion beam during the transfer (Figures 1(h-i)). Data from a heating experiment on a cross-sectional specimen of an Ag-thin film on (100) Si substrate (Figure 2) is presented to demonstrate the quality of the specimens that can be prepared using this approach for in situ experiments [4].

\section{References:}

[1] Jungjohann, K., Carter, C.B. “Transmission Electron Microscopy: Diffraction Imaging \& Spectrometry, Carter, C.B. \& Williams, D.B (Eds.), (Springer, New York) p.17.

[2] X. Zhong et al, Microsc. Microanal, 22 (2016), p.1350.

[3] M. Duchamp et al, Microsc. Microanal, 20 (2014), p.1638.

[4] This work was supported in part by a research grant from FEI Company under an FEI-UConn partnership agreement. The studies were performed in the UConn/FEI Center for Advanced Microscopy and Materials Analysis (CAMMA). 

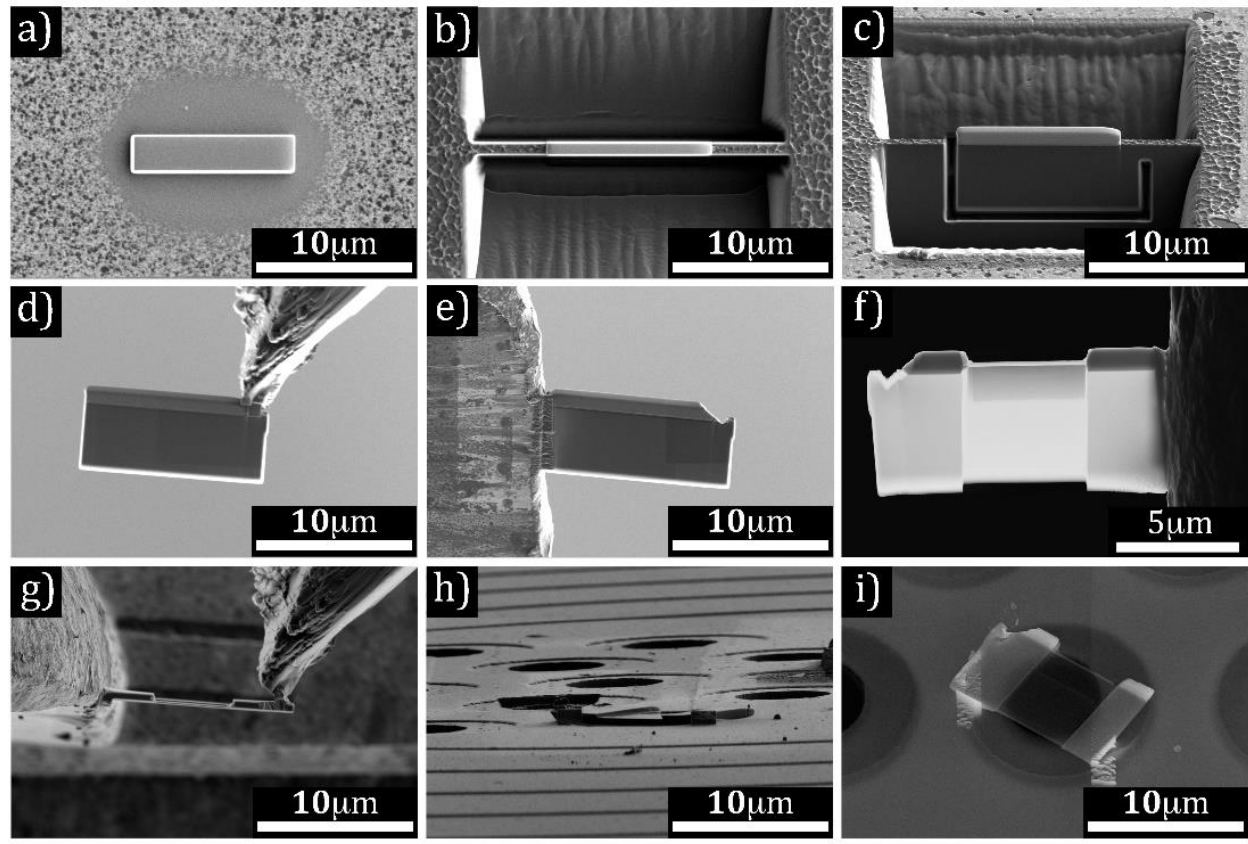

Figure 1. Sequence of operations showing: (a) Pt cap, (b) trench milling, (c) U-cut to separate lamella from bulk sample, (d) lamella separated from bulk sample, (e) lamella attached to Cu grid on flip stage, (f) HAADF STEM image of lamella after final thinning, (g) nanomanipulator attached to free end of specimen before separation from $\mathrm{Cu}$ grid on flip stage, (h-i) secondary electron micrographs of specimen on the MEMS chip obtained using the ion and electron columns, respectively.

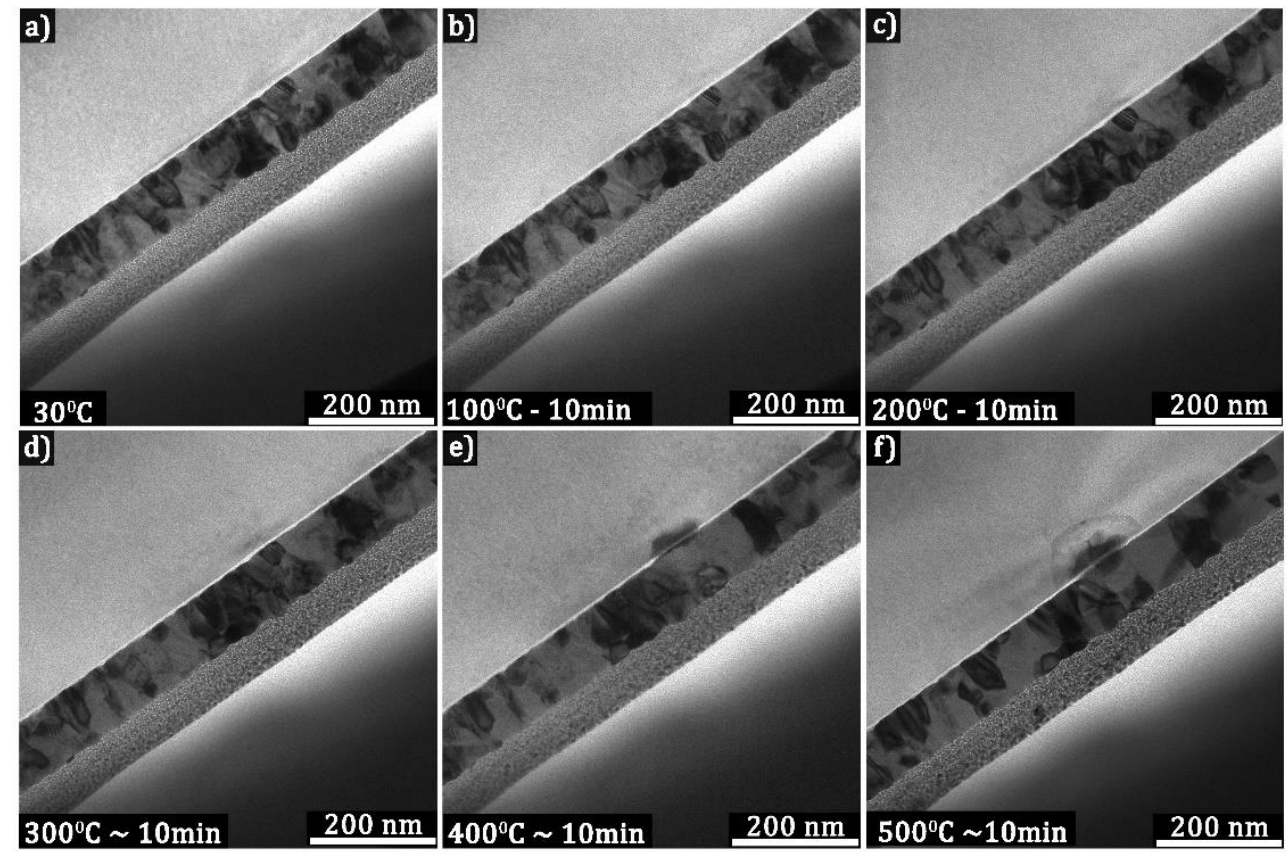

Figure 2. BF TEM images from Ag on (100) Si cross sectional TEM samples obtained after 10 minute isothermal holds at: (a) $30^{\circ} \mathrm{C}$, (b) $100^{\circ} \mathrm{C}$, (c) $200^{\circ} \mathrm{C}$, (d) $300^{\circ} \mathrm{C}$, (e) $400^{\circ} \mathrm{C}$, (f) $500^{\circ} \mathrm{C}$. 